\title{
Oral Lubrication Matters: Effects on Satiety
}

Emma M. Krop ${ }^{a}$, Marion M. Hetherington ${ }^{b \dagger}$, Sophie Miquel ${ }^{c}$, Anwesha Sarkar ${ }^{a^{*}}$

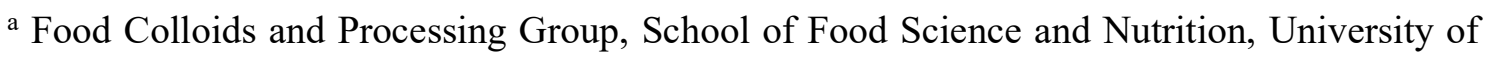
Leeds, Leeds LS2 9JT, United Kingdom

${ }^{\mathrm{b}}$ School of Psychology, University of Leeds, Leeds LS2 9JT, United Kingdom

${ }^{\mathrm{c}}$ Mars Wrigley Confectionery, 1132 West Blackhawk Street, Chicago, IL 60642, USA

\section{Corresponding authors:}

$†$ Professor Marion Hetherington

School of Psychology, University of Leeds, Leeds LS2 9JT, UK

E-mail address: M.Hetherington@leeds.ac.uk

Phone: +44 (0) 1133438472

*Dr Anwesha Sarkar

Food Colloids and Processing Group,

School of Food Science and Nutrition, University of Leeds, Leeds LS2 9JT, UK

E-mail address: A.Sarkar@leeds.ac.uk

Phone: +44 (0) 1133432748 


\begin{abstract}
As overeating, overweight and obesity remain public health concerns, it is crucial to design satiety-enhancing foods that suppress appetite and lower snack intake. Existing research identifies oro-sensory targets to promote satiation and satiety within the "satiety cascade", yet it remains unclear as to whether it is 'chewing' or 'oral lubrication' that might amplify satiation signals. Here we have combined techniques from experimental psychology, food material science and mechanical engineering to measure the role of chewing and lubrication using novel, model foods as preloads on subjective appetite and intake of a salty snack. Three mint flavoured hydrogels were engineered to vary in their texture (fracture stress) and lubrication (inverse of friction coefficient) properties, and a control group received a mint tea. Results showed that snack intake was suppressed by $32 \%$ after eating the low chewing/high lubricating preload as compared to the high chewing/low lubricating preload $(p<0.05)$. No other significant effects were found for snack intake. Hunger ratings decreased from $t_{1}$ to $t_{3}(p<0.05)$, however differences between conditions were subtle and not significant. Thus, this proof-of-concept study demonstrates that manipulating oral lubrication is a promising new construct to reduce snack intake that merits future research in the oro-sensory satiety domain.
\end{abstract}


The global occurrence of overweight (body mass index, BMI, $25-<30 \mathrm{~kg} / \mathrm{m}^{2}$ ) and obesity $\left(\mathrm{BMI} \geq 30 \mathrm{~kg} / \mathrm{m}^{2}\right)$ is escalating at an unprecedented rate, which is associated with substantial morbidity and mortality consequences. ${ }^{5}$ WHO (2016) estimated about 1.9 billion adults being overweight worldwide with $>30 \%$ among them being obese. ${ }^{6}$ Overweight and obesity are caused by a chronic imbalance between energy intake and expenditure; and so in order to prevent overeating relative to energy needs, there has been an upsurge in research efforts to design satiation- and satiety-enhancing foods that suppress appetite and prevent overconsumption. Satiation has been defined as the processes leading to the termination of an eating event, and satiety as the inhibition of appetite and eating, as understood within the multifactorial concept of the 'satiety cascade'. ${ }^{1,2,7-9}$ Both satiation and satiety responses contribute to the termination of energy intake, and therefore understanding their contributing factors is pertinent for designing food-based approaches to limit overeating with potential in the longer term to influence weight management. ${ }^{10}$

The role of cephalic phase responses, i.e. anticipatory physiological preparation for digestion, absorption, gut hormonal triggers of humans and other primates has been pioneered by the seminal work of Pavlov more than a century ago. ${ }^{11}$ It is now well recognized in humans that when food is infused directly into the gastrointestinal tract, thus bypassing the oral cavity, that the satiation response is significantly lower than during normal oral consumption of the same food $^{12}$, whereas using sham-feeding similar satiation responses are obtained compared to normal food ingestion. ${ }^{13}$ Although the role of oral processing on satiation and satiety has been well established, the quantitative understanding of which dimensions of oral processing influence this has remained elusive. ${ }^{3,14-18}$ Based on a recent systematic review and metaanalysis on relating oral processing to satiety, it was demonstrated that extending the orosensory exposure time to foods leads to a significant reduction in self-reported hunger $(-0.20$ effect size, $95 \%$ confidence interval CI: $-0.30,-0.11)$ and food intake $(-0.28$ effect size, $95 \%$ 
CI: $-0.36,-0.19) .{ }^{3}$ Interestingly, in many if not most of these satiety trials involving oro-sensory cues, 'food rheology' (i.e. liquid versus solid foods, texture/thickness manipulations) has been used as a 'gold standard'-design tool to influence the number of chews, oral residence time or eating rate, and thus, impact satiety outputs such as appetite ratings (hunger desire to eat), food intake and gut hormonal release. ${ }^{14,17,19-21}$ However, during oral exposure, food characteristics change dramatically due to lubrication by saliva as well as the saliva-food mixture that might coat the tongue and other oral surfaces that are of fundamental importance for deglutition and satisfaction. ${ }^{22,23}$ Although oral lubrication or friction provided by food is a crucial aspect of this fundamental biological process occurring in the mouth, its' mechanistic effects on psychological and physiological consequences implicated in altering the motivation to eat remain under-researched. ${ }^{3}$

\section{Objectives}

The present study was designed to address this fundamental knowledge gap using a crossdisciplinary approach by combining techniques from experimental psychology, food material science and mechanical engineering. Here we report the effect of novel preload 'biopolymeric hydrogels' on appetite ratings and food intake from both the 'chewing' and 'oral lubrication' perspective, of which the latter has never been used as a construct in satiety trials. The selected hydrogels had no energy content and varied in texture in two specific domains: the chewing as well as the lubrication properties. The chewing properties of the hydrogels were measured by instrumental texture analysis as well as video recording of the oral processing behaviour, whereas oral lubrication was defined as a reduction in coefficient of friction using tribology, a technique translated from mechanical engineering (see Figure 1a). The main objective was to investigate which food design factor between chewing and lubrication might lower snack intake, and whether this is reflected in subjective appetite. The second objective was to study 
whether the hydrogel preload effects were variable according to eating context (eating alone or in a group).
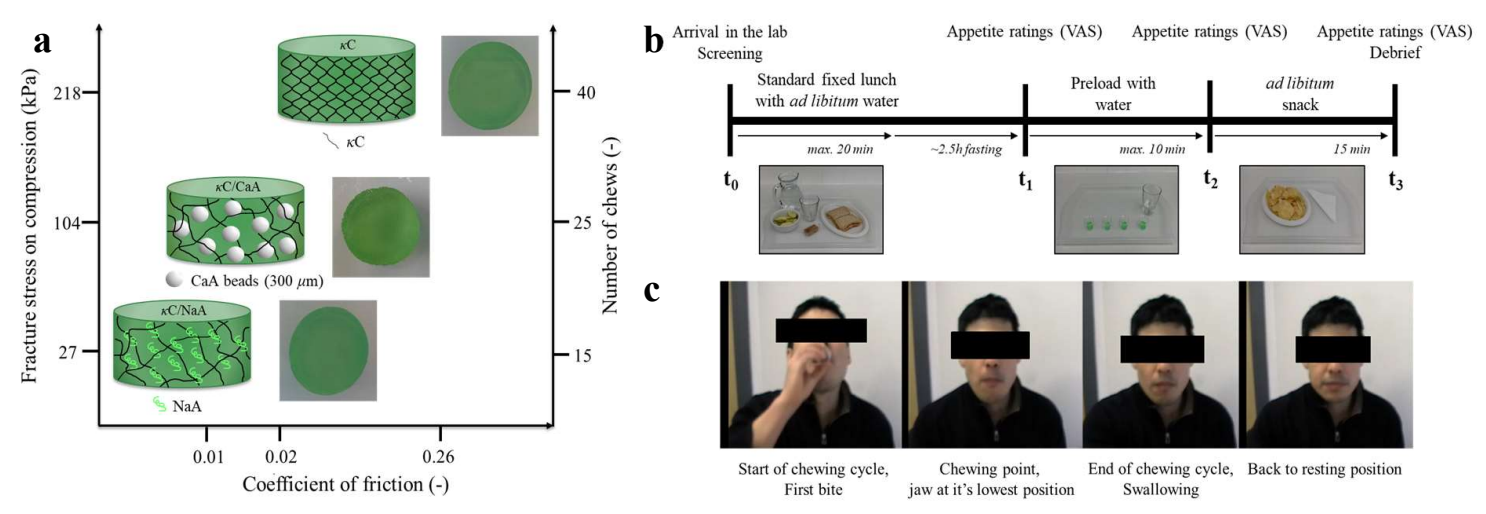

Figure 1. Schematic representation of the three different preload gels made up of a $\kappa$ carrageenan $(\kappa \mathrm{C})$ gel matrix alone or with the addition of sodium alginate $(\mathrm{NaA})$ or calcium alginate beads $(\mathrm{CaA})$ to create distinct chewing and lubrication properties (a). These properties were based on instrumental characterisation by texture analysis (fracture properties) and tribology (coefficient of friction is a measure of the reverse of lubrication), as well as characterisation of the oral processing behaviour using video analysis. Timeline and study procedures of each experimental phase with appetite ratings scored on Visual Analogue Scales (VAS) as a function of time (b). Frame-by-frame video analysis of oral processing behaviour (eating duration from first bite to swallowing and number of chews) (c).

\section{RESULTS}

\section{Participants' characteristics}

Fifty-nine participants completed the study. Before the start of the study, the participants' liking and their preparedness to eat an example of the novel preload foods $(3 \kappa \mathrm{C}$ gel $)$ used in this study were tested. The mean liking for the test food was $34.7 \pm 23.1 \mathrm{~mm}$ and all participants indicated they were willing to eat the model foods as part of this study (except four participants who received the control sample). After data collection was completed, four participants were excluded from the analysis due to the following reasons - three participants ate less than $12.5 \mathrm{~g}$ 
of the snack, which is half the size of a normal portion, meaning these participants were considered not to truly have eaten a snack; and one participant consumed all of the provided snack, and thus, exhibited the 'cleaning the plate effect', indicating the snack was not truly ad libitum. Thus, the data for 55 participants (16 male, 39 female) were analysed (see Table 1). Participants ranged in age from 18 to 45 year (mean 26.3, SEM 1.0 years) and BMI from 17.7 to 33.1 (mean 23.0, SEM $0.4 \mathrm{~kg} / \mathrm{m}^{2}$ ). Most of the participants had a BMI score in the normal range $(n=40)$, but three were classified as underweight, nine were overweight and three were obese. Eating restraint scores on the DEBQ showed that three males $(>2.89)$ and six females $(>3.39)$ were restrained eaters ${ }^{24}$, with a mean score of $2.17 \pm 0.17$ for males and $2.59 \pm 0.10$ for females. Most of the participants were students at the University of Leeds $(n=48)$, and seven participants were employed.

Table 1. Number of participants in the different preload conditions.

\begin{tabular}{l||ccccc} 
& $\mathbf{3} \boldsymbol{\kappa} \mathbf{C}$ & $\mathbf{1 . 5} \boldsymbol{\kappa} \mathbf{C 0 . 5 N a A}$ & $\mathbf{2 . 4} \boldsymbol{\kappa} \mathbf{C 0 . 2 C a A} \mathbf{3 0 0}$ & Mint tea & Total \\
\hline \hline Total & 13 & 13 & 15 & 14 & 55 \\
\hline Male & 5 & 3 & 3 & 5 & 16 \\
Female & 8 & 10 & 12 & 9 & 39 \\
\hline - Individual & 8 & 7 & 10 & 9 & 34 \\
\hline Male & 5 & 2 & 2 & 3 & 12 \\
Female & 3 & 5 & 8 & 6 & 22 \\
\hline - Group & 5 & 6 & 5 & 5 & 21 \\
\hline Male & 0 & 1 & 1 & 2 & 4 \\
Female & 5 & 5 & 4 & 3 & 17 \\
\hline
\end{tabular}

\section{Effect of oral processing on snack intake}

The amount of snack was significantly different after the four preload conditions $(p<0.01)$, with the snack intake being supressed by $32 \%$ after the soft/high lubricating mint stimulus $(1.5 \kappa \mathrm{C} 0.5 \mathrm{NaA}, 36.7 \pm 2.9 \mathrm{~g})$ compared to the hard/low lubricating stimulus $(3 \kappa \mathrm{C}, 59.1 \pm 6.4$ g), see Figure 2a. The overall snack intake also differed between session types $(p<0.01)$, with 
the intake in the group sessions being higher $(58.4 \pm 4.3 \mathrm{~g})$ than when eating alone $(44.9 \pm 3.3$ g), as was expected due to the social setting. Figure $\mathbf{2 b}$ shows the difference in snack intake between conditions separated by session type (alone or in a group). No interaction effects were found between condition and session type $(p=0.604)$. While there was no significant effect of condition for the participants eating alone $(p=0.463)$, there were significant differences in snack intake between preloads when participants ate in a group setting (see Figure 2b). This suggests that although the effect of preload was small, it was revealed due to either the larger sample size or the general increase in food intake during social eating. Also, the effect of gender was analysed but main effects and interactions were not significant, as consistent with previous research ${ }^{16}$, therefore all subsequent analyses were reported for the group as a whole: male and female, and individual and group sessions together.
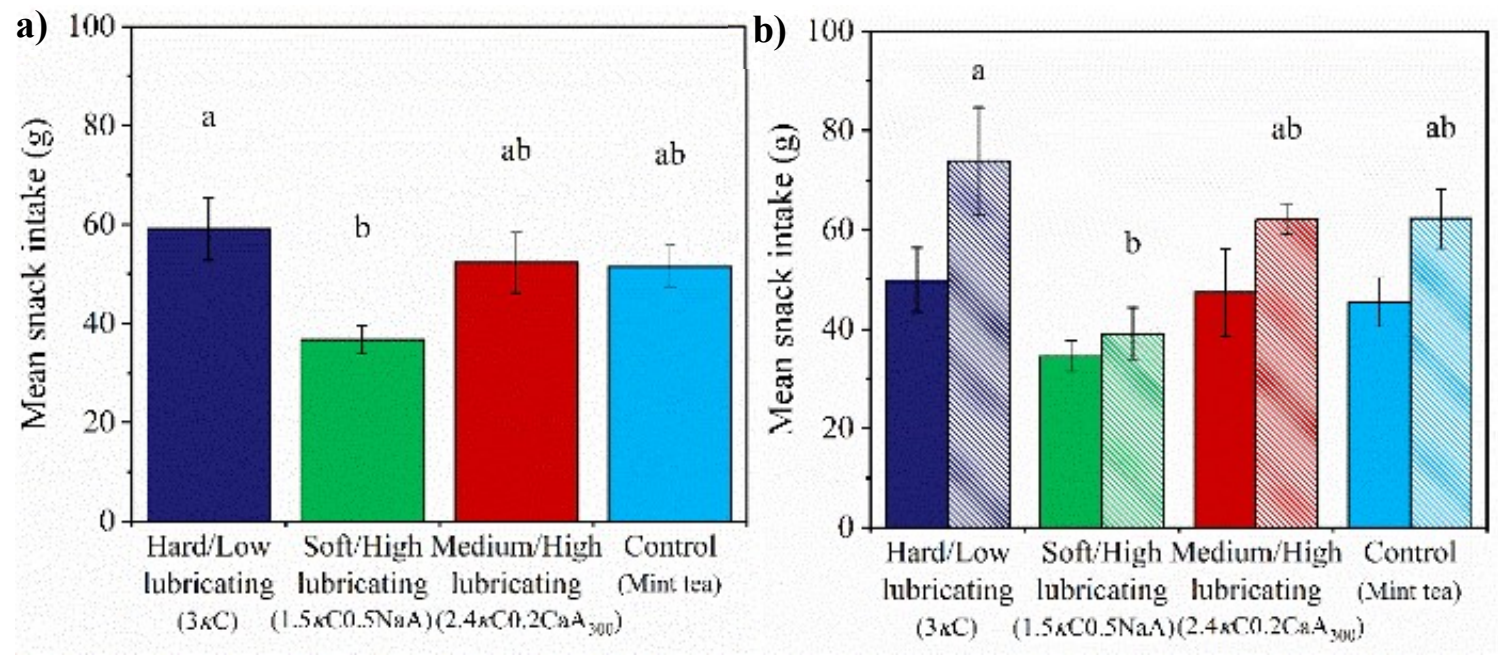

Figure 2. Mean ( \pm SEM) snack intake after preload (a) and snack intake split between the individual sessions (solid fill, $\mathrm{n}=34$ ) and group sessions (diagonal lines, $\mathrm{n}=21$ ) (b) after the four preload conditions: $3 \kappa \mathrm{C}(\mathbf{\square}), 1.5 \kappa \mathrm{C} 0.5 \mathrm{NaA}(\mathbf{\square}), 2.4 \kappa \mathrm{C} 0.2 \mathrm{CaA}_{300}(\mathbf{\square})$ and mint tea $(\mathbf{\square})$. There is no significant effect of condition for the individual sessions, nor is there an effect of session type within conditions, but there is an effect of condition for the group sessions $(p<$ 
0.05). Different lower case letters indicate statistically significant differences between conditions $(p<0.05)$.

\section{Effect of oral processing on subjective appetite ratings}

Hunger ratings did not differ by condition, nor was there a significant condition by time interaction. However, the hunger ratings did change over the different time points $\left(t_{1}-t_{3}\right.$, see Figure 3a), with a significant decrease over time $(\mathrm{F}(2,102)=14.87, p<0.001)$. Post-hoc tests revealed that post-snack hunger at $t_{3}$ was significantly lower than at $t_{1}$ and $t_{2}$. There was no significant difference between ratings at $t_{1}$ and $t_{2}$. Similar effects were found for desire to eat $(\mathrm{F}(2,102)=14.15, p<0.001)$ and appetite $(\mathrm{F}(2,102)=14.34, p<0.001)$, see Supplementary

Figures 1a and 1b. The fullness ratings mirrored those of the hunger, desire to eat and appetite ratings showing a significant time effect $(\mathrm{F}(2,102)=11.97, p<0.001)$, where $\mathrm{t}_{3}$ hunger was significantly higher than $t_{1}$ and $t_{2}$ fullness ratings (see Figure $3 \mathbf{b}$ ).

There was no significant effect of condition on thirst ratings (Figure 3c), nor was there any interaction effect of condition by time. However, there was an effect of time alone $(F(2,96)=$ 31.62, $p<0.001)$. Post hoc tests revealed that post-snack thirst was higher than pre-preload or post-preload. Thirst ratings were also lower post-preload compared the pre-preload at the start of the second session. There were no interaction effects between conditions and time points, and there was no effect of condition on desire to eat something sweet or desire to eat something salty. However, desire to eat something sweet $(\mathrm{F}(2,96)=4.52, p<0.05)$ and desire to eat something salty $(\mathrm{F}(2,96)=33.28, p<0.001)$ did significantly change over time (Supplementary Figures 1c and 1d). To make sure none of the preloads invoked a stronger feeling of nausea, due to the novelty of the model foods or the presence of the hydrocolloids in the preloads, nausea was rated over time as well (Figure 3d). There was no significant main effect of preload condition or time point, nor was there any interaction effect of condition vs time. 

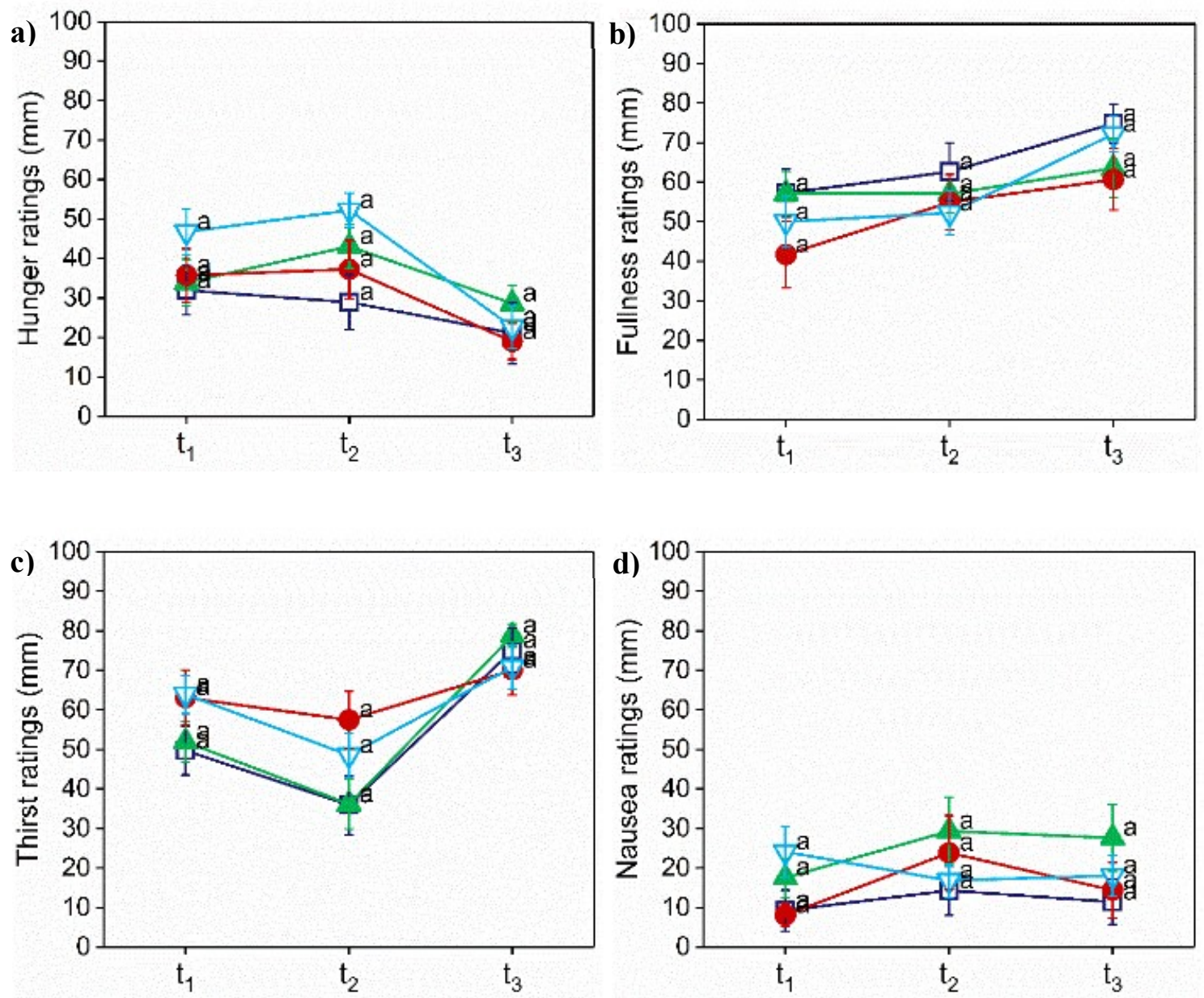

Figure 3. Mean ( \pm SEM) hunger (a), fullness (b), thirst (c) and nausea (d) ratings over time for the four preload conditions: $3 \kappa \mathrm{C}(\square), 1.5 \kappa \mathrm{C} 0.5 \mathrm{NaA}(\boldsymbol{\Delta}), 2.4 \kappa \mathrm{C} 0.2 \mathrm{CaA}_{300}(\boldsymbol{\bullet})$ and mint tea $(\nabla)$. Different lower case letters indicate statistically significant differences between conditions $(p<0.05)$.

\section{Perception of the study foods}

The pleasantness, strength of the mint flavour, sweetness or chewiness of the preload foods were rated on $100 \mathrm{~mm}$ VAS. One-way ANOVA indicated that pleasantness, mint flavour and sweetness did not differ between the preload conditions. However, the chewiness of the preload samples was significantly different $(\mathrm{F}(3,51)=31.30, p<0.001)$. The post hoc test indicated that the mint tea (control sample) was not perceived as chewy at all (mean 3.1, SEM 1.8), the 
$1.5 \kappa \mathrm{C} 0.5 \mathrm{NaA}$ was significantly more chewy (mean 37.3 , SEM 8.3 ) than the mint tea, and $3 \kappa \mathrm{C}$ (mean 77.4, SEM 6.5) and $2.4 \kappa \mathrm{C} 0.2 \mathrm{CaA}_{300}$ (mean 67.2, SEM 5.9) were the most chewy.

The desire to eat (mean 64.3, SEM .13) and the pleasantness of the crisps (mean 75.7, SEM 2.2) were evaluated after a first bite of the crisps, and they did not differ significantly between conditions. The sweetness (mean 17.1, SEM 2.4) and saltiness (mean 74.1, SEM 2.2) of the crisps were also rated similarly after a normal size snack during the different conditions, showing no significant differences.

\section{Correlations}

Pearson correlations between chewiness of the preload and the snack intake showed that they were not related $(\mathrm{r}=0.056, p=0.687)$. Food intake between the different preload gels also did not correlate with the perceived pleasantness $(\mathrm{r}=-0.132, p=0.338)$ or any potential induced nausea after eating the preload foods $(\mathrm{r}=-0.189, p=0.168)$.

\section{DISCUSSION}

The present study investigated whether model hydrogels with varying chewing and oral lubrication properties had a significant influence on self-reported appetite measures, such as hunger, fullness and desire to eat, as well as the intake of a snack. It was hypothesized that more chewing would lead to lower food intake, as reported in previous studies. ${ }^{3}$ Interestingly, results found that snack intake was only lowered after the consumption of the soft/high lubricating preload sample $(1.5 \kappa \mathrm{C} 0.5 \mathrm{NaA})$ compared to the hard/low lubricating preload $(3 \kappa \mathrm{C})$, but not after the control, suggesting that not the chewing but the lubricating properties governed subsequent intake of a salty snack. Sensory ratings for the different preloads did not reveal a significant difference in terms of pleasantness, strength of mint flavour or sweetness, and therefore these characteristics could not account for the suppressed food intake after the soft/high lubricating preload $(1.5 \kappa \mathrm{C} 0.5 \mathrm{NaA})$. Nevertheless, there was no significant difference 
between the soft/high lubricating preload $(1.5 \kappa \mathrm{C} 0.5 \mathrm{NaA})$ and medium/high lubricating preload $\left(2.4 \kappa \mathrm{C} 0.2 \mathrm{CaA}_{300}\right)$ or the control tea sample. Therefore, it seems unlikely that the lubrication alone was responsible for the lower snack intake in the soft/high lubricating preload $(1.5 \kappa \mathrm{C} 0.5 \mathrm{NaA})$. Previous research analysing the hydrogel preloads using a sensory panel found that the soft/high lubricating preload $(1.5 \kappa \mathrm{C} 0.5 \mathrm{NaA})$ was rated more 'pasty' compared to the medium/high lubricating preload $\left(2.4 \kappa \mathrm{C} 0.2 \mathrm{CaA}_{300}\right)$, though they were rated similarly for other indexes of lubrication. ${ }^{4}$ This pastiness was defined as 'a sensation of the presence of wet/soft (immiscible) solids in the mouth', which could result in a certain amount of mouth coating. Such mouth coating aspects of alginate have previously been reported as related to a mouth moistening and hydrating property ${ }^{25,26}$, which in turn might lead to a lower snack intake. To make sure this mouth coating did not lead to any feelings of nausea, the nausea ratings were checked, however no significant differences were found between conditions after the consumption of the preloads.

Snack intake after the hard/low lubricating $(3 \kappa \mathrm{C})$ and the control sample (no chewing/low lubricating) did not show a significant difference either, indicating that it was not the chewing properties that determined snack intake after the preload. This is inconsistent with previous research, which showed that higher level of chewing did indeed reduce food intake. ${ }^{3,14,18}$ This might be explained by the short exposure time of 10 minutes and the low amount of elicited chewing in this period, indicating that the total chewing time may not have been sufficiently long enough to influence food intake. Future research incorporating more hydrogel pieces into the preload to increase overall chewing time may find a more pronounced effect on food intake. However, there are other studies that confirm no impact of chewing on food intake. ${ }^{27}$

The effect size was considered relatively small, which is also consistent with previous research investigating oro-sensory stimulation. ${ }^{16}$ This may be related to a small effect of chewing or lubrication during oral processing, or the amount of preload gels (four or five units per 
participant) was rather small. The novelty of the preload hydrogels and their generally low rated pleasantness were a consideration in providing a limited amount of the preload foods ${ }^{28}$, as well as not wanting to prevent any further food intake due to the volume of the preload. The preload hydrogels used in this study were based on their differences in chewing and oral lubrication properties, however they were not perfectly matched. No samples with high chewing and high lubricating properties were tested. In addition, the in-vivo oral lubrication effects of the preloads, i.e. the lubrication contributed by the bio-lubricant saliva (internal) versus hydrogels (external), were not checked whereas chewing properties were by video analysis of the chewing behaviour.

The present study also found that preload foods with varying chewing and oral lubrication properties did not significantly influence self-reported appetite measures, such as hunger, fullness and desire to eat, indicating that one preload did not lead to higher or lower selfreported appetite ratings than any of the other preloads. In addition, a decrease in hunger, desire to eat, appetite and desire to eat something salty, and an increase in fullness ratings were observed over time in the following snack intake in all preload conditions. Thus, this confirmed that the participants consumed the snack until satiety was reached.

The use of ready salted crisps as a salty snack in the current study may have influenced the results. Cravings and liking for the crisps may have overshadowed the chewing and oral lubrication effects of the preloads. Also, no alternative salty snacks or sweet snacks were presented to study their effects. The lack of effect on snack intake may also be due to individual differences in participants' usual snacking behaviour, and suggests that if sweet snacks had also been provided, clearer differences may have been found. Conversely, increasing variety by providing both salty and sweet snacks might increase intake ${ }^{29}$, and thus, might overpower any effects due to the preloads. 
Snack intake was greater in a group setting compared to eating alone, also confirming the hypothesis that social interactions during a snack increases food intake. ${ }^{30}$ An independent between-subjects design was used in the present study to facilitate easier panel recruitment and flexibility. Better results might have been obtained with a within-subjects design where the random noise would be minimized. ${ }^{31}$ However, a within-subjects design would have resulted in increased familiarity with the preload hydrogels, and would be associated with increased expected satiation and satiety.

The aim of this study was to investigate whether chewing and lubrication during oral processing, manipulated by hydrogel preload foods, had an influence on snack intake and selfreported appetite ratings. Results from this proof-of-concept study demonstrated that snack intake was reduced in soft/high lubricating preloads, but not in hard/low lubricating, medium/high lubricating or a liquid, low lubricating control samples. The mechanism by which oral lubrication rather than chewing played a prominent role in reducing subsequent food intake of a salty snack was associated with the 'mouth-coating' aspects of the preload; however, exact biological cross-talk between mouth-coating, tactile perception and mechano-receptor stimulated satiation demands future systematic studies.

\section{METHODS}

\section{Participants}

The study was performed at the University of Leeds, UK. Participants were recruited using a poster campaign around the university campus, departmental recruitment emails and emails sent to a database with people who signed up voluntarily with an interest in participating in human studies. Healthy male and female volunteers were eligible for the study, aged between 18-55 years, without any dental deficiencies or problems with chewing or swallowing, that did not have any food allergies or intolerances to the used study foods and were not taking any medications that might influence appetite or food intake. The experimental protocol of this 
study was approved by the University of Leeds, School of Psychology Research Ethics Committee (reference number PSC-190) and all participants signed informed consent before their participation. Participants were not told of the exact aim of the study, instead they were told that the aim of the study was to investigate the effect of a mint stimulus on their perception of a salty snack. Students from the School of Psychology were awarded course credits for their participation, while other participants were entered into a prize draw with three participants being randomly selected to win a $£ 10$ shopping voucher as compensation.

\section{Experimental design}

The study followed a randomized between-subjects design where participants were randomly assigned to one of four conditions. In the different conditions, participants received one of four preload foods (model hydrogels) with different chewing and oral lubrication properties, as based on instrumental characterisation ${ }^{4}$ and oral processing behaviour analysis. The preloads were similar in palatability and mint flavour intensity, and contained less than one kcal. To study the effect of social interactions and to facilitate a larger number of participants, testing sessions took place either individually or in groups of five to six people.

\section{Study foods}

A standardised lunch with fixed amounts was given to all participants prior to the start of the study. The lunch consisted of a cheese sandwich, apple, an oatmeal flapjack and ad libitum water. The sandwich was prepared using two slices $(186 \mathrm{kcal} / 80 \mathrm{~g})$ of Kingsmill medium sliced 50/50 bread (Allied Bakeries, UK), $12 \mathrm{~g}$ (84 kcal) Flora buttery margarine (Unilever, UK) and $32 \mathrm{~g}$ (133 kcal) grated British medium cheddar cheese. A Braeburn apple was washed and cut in slices. A $100 \mathrm{~g}(47 \mathrm{kcal})$ was weighed out, and presented with the sandwich and an individually wrapped flapjack slice $(159 \mathrm{kcal} / 37 \mathrm{~g})$ (see Figure 1b). All products were purchased at a local supermarket. Participants were instructed to consume all the foods 
provided, containing $609 \mathrm{kcal}$ in total. For the ad libitum snack, ready salted crisps (Walkers Snack Foods Ltd., UK) were provided (526 kcal/100 g).

For the preloads, novel mint flavoured hydrogels were selected based on their different chewing and lubrication aspects as characterised in our previous work ${ }^{4}$, see Figure 1a. The differences in chewing and lubrication were achieved by varying the concentration of different gelling agents, i.e. $\kappa$-carrageenan $(\kappa \mathrm{C})$ and sodium alginate $(\mathrm{NaA})$, or by introducing calcium alginate beads $(\mathrm{CaA})$ to create a level of inhomogeneity. The $3 \kappa \mathrm{C}$ gel represents a $3 \mathrm{wt} \% \kappa$ carrageenan gel with high chewing and low oral lubricating properties; the $1.5 \kappa \mathrm{C} 0.5 \mathrm{NaA}$ sample represents a mixed $1.5 \mathrm{wt} \% \kappa$-carrageenan and $0.5 \mathrm{wt} \%$ Na-alginate gel with low chewing and high lubricating properties; and the $2.4 \kappa \mathrm{C} 0.2 \mathrm{CaA}_{300}$ denotes the $2.4 \mathrm{wt} \% \kappa$ carrageenan with a layer of $0.2 \mathrm{wt} \%$ Ca-alginate beads, $300 \mu \mathrm{m}$ in diameter, with medium chewing and high lubricating properties. ${ }^{4}$ The hydrogels were unsweetened, but flavoured with peppermint aroma and coloured with green food colouring to increase acceptability. The samples were presented in bite-size round pieces (diameter $25 \mathrm{~mm}$, height $10 \mathrm{~mm}$ ) in small, shot-glass type plastic cups. Peppermint tea (Pure Peppermint, Twinings, UK), purchased at a local supermarket and coloured with the same food colouring as for the gels, was used as a liquid control sample that did not require any chewing. The tea was presented in the same cups as the gels, filled up to the same height as the gels.

\section{Instrumental characterisation of the hydrogels}

The instrumental properties of the gels were characterised as related to the chewing and the lubrication aspects using texture analysis and tribology, respectively. Further details on the methodology and results have been published elsewhere. ${ }^{4}$

Uniaxial single compression tests were performed on the gels with a TA-TX2 Texture Analyzer Micro Systems Ltd., Surrey, UK) using a cylindrical probe (diameter $59 \mathrm{~mm}$ ), attached with a 
$50 \mathrm{~kg}$ load cell. The tests were carried out at room temperature at a constant speed of $1 \mathrm{~mm} / \mathrm{s}$ and the deformation level was set at $80 \%$ strain. Measurements were done in triplicate on at least four different preparation days, and mean values of fracture stress were calculated using:

$$
\text { Fracture stress }(P a)=\frac{\text { Force }_{t}(N)}{A_{t}\left(m^{2}\right)}
$$

where Force $\mathrm{t}_{\mathrm{t}}$ and $\mathrm{A}_{\mathrm{t}}$, the cross-sectional area of the compressed sample at fracture, are determined over time at the fracture point.

Tribology measurements were performed on the hydrogels after simulated oral processing in presence of artificial saliva ${ }^{32}$ using a Mini Traction Machine (MTM2, PCS Instruments, London, UK). The smooth steel surfaces in this device, commonly used in engineering disciplines, were replaced by a polydimethylsiloxane (PDMS) ball and disc set-up at $37{ }^{\circ} \mathrm{C}$ to mimic the oral surfaces (surface roughness, $R_{a}<50 \mathrm{~nm}$ ). ${ }^{33,34}$ The rolling speed was reduced from 1000 to $1 \mathrm{~mm} / \mathrm{s}$ at a load of $2 \mathrm{~N}$, using a slide-to-roll ratio (SRR) of $50 \%$, and the coefficient of friction in the mixed lubrication regime $(50 \mathrm{~mm} / \mathrm{s})$ was measured in triplicate.

\section{Study procedure}

A schematic overview of the timeline and study procedure can be found in Figure 1b. On the day of testing, participants were instructed to eat their normal breakfast and then attend the lab at lunchtime between 12:00-13:00 h. As a compliance measure, participants were asked about the last time they had anything to eat and drink, and what they ate/drank. All participants were asked to fill out a demographics questionnaire, inquiring about things such as age, self-reported body mass index (BMI), health and dietary preferences. The questionnaire also tested for eating restraint using the Dutch Eating Behaviour Questionnaire (DEBQ). ${ }^{24}$ Then, participants were provided with an example of the novel preloads used in this study $(3 \kappa \mathrm{C}$ gel), and were asked for their liking and preparedness to eat similar stimuli for the purposes of this study. In addition, liking for the commercially available test foods was checked. A fixed lunch was then served to 
each participant to control for hunger (Figure 1b), and panellists were asked to return to the lab $3 \mathrm{~h}$ after the lunch for the snack.

Participants were instructed not to eat or drink anything besides water between sessions. As a compliance measure, participants were asked about the last moment they ate or drank anything at the start of the second session. Next, participants completed the pre-preload $\left(\mathrm{t}_{1}\right)$ appetite questionnaire, by rating their level of hunger, fullness, desire to eat, appetite, thirst, nausea, desire to eat something sweet and desire to eat something salty on a $100 \mathrm{~mm}$ visual analogue scale (VAS), anchored from 'not at all' to 'extremely'. After filling out their appetite ratings, participants were offered the preload stimuli and $50 \mathrm{~mL}$ of water. Males received five units and females four units to account for the difference in energy requirements between men and women. Participants were instructed to consume the first mint stimulus followed by a sip of water until all the mint stimuli and the water were consumed within 10 minutes. Afterwards, the perceptions of the mint stimulus were evaluated (VAS ratings), followed by another appetite questionnaire $\left(\mathrm{t}_{2}\right)$. Then, the participants were offered a snack of $100 \mathrm{~g}$ ad libitum ready salted crisps (pre-weighed amount, $526 \mathrm{kcal}$ ), as shown in Figure 1b. To distract the participants form the true nature of the study, they rated their desire to eat and pleasantness of the crisps after a first bite. After that, participants were instructed to eat a normal sized snack, eat as much as they liked within 15 minutes until they felt comfortably full, and to rate their sensory perception of the crisps. Participants were asked not to use their phone within this 15minute period. Immediately after the snack, participants re-rated appetite $\left(\mathrm{t}_{3}\right)$ and answered a final debrief questionnaire, which invited participants to consider the true purpose of the study and to check participants' experience with the novel preload foods or similar food products.

\section{Oral processing characteristics}

To analyse the eating behaviour and make sure participants properly followed the study protocol, a small selection of the participants were asked permission to video record them while 
eating the preload model food $(\mathrm{n}=21)$. A digital camera (Panasonic SDR-H90) on a tripod was positioned in front of the participant, and participants were instructed to look straight into the camera while eating the preloads but were permitted to take short breaks in between samples if needed. Videos were analysed using the software The Observer XT 12 (v 12.5, The Noldus Information Technology bv, The Netherlands). A coding scheme was created to analyse certain chewing behaviours including number of chews and eating duration adapted from previous studies ${ }^{35,36}$, see Figure 1c. A chew was defined as the point event the jaw was at the lowest level during a masticatory cycle (closing action). Eating duration was defined as the time between first bite and swallowing, identified as the first main swallow of one of the preload samples at the end of the rhythmic rotary chewing movements. From these characteristics, the chewing frequency could be calculated by dividing the number of chews by the total eating duration. ${ }^{36-38}$

\section{Statistical analysis}

All statistical analyses were performed using SPSS (IBM ${ }^{\circledR}$ SPSS ${ }^{\circledR}$ Statistics, v24, SPSS Inc, Chicago, USA). Results are presented as mean \pm standard error of mean (SEM), and significance level was set at $\mathrm{p}<0.05$ (2-tailed), unless stated otherwise. Differences between conditions were tested by independent factorial analysis of variance (ANOVA) for food intake and repeated measures to assess condition effects on appetite ratings, followed if appropriate by a post-hoc Bonferroni correction for multiple comparisons. Pearson's product moment correlations were calculated to assess the relationship between the different preload conditions and perceived chewiness and hunger ratings at the three different time points.

\section{Acknowledgements}

We would like to thank Hannah Clark for her help in recruiting panellists and running the satiety experiment. This work was supported by the University of Leeds 110 Anniversary 
Research Scholarship with matched support from Mars Wrigley Confectionery, awarded to EK for her PhD studies.

\section{Author contributions}

The authors' responsibilities were as follows - MMH and AS: designed the research; EK: developed the experimental protocol, conducted the satiety trial and analysed the data; EK: wrote the manuscript; AS and MMH: contributed to revisions of the manuscript; AS and MH: had primary responsibility for final content; and all authors: read, edited and approved the final manuscript.

\section{Competing interests}

None.

\section{REFERENCES}

1 Blundell, J. Making claims: functional foods for managing appetite and weight. Nature Reviews Endocrinology 6, 53, doi:10.1038/nrendo.2009.224 (2010).

2 Blundell, J. E., Rogers, P. J. \& Hill, A. J. in Food Acceptance and Nutrition 205-219 (1987).

3 Krop, E. M. et al. Influence of oral processing on appetite and food intake - A systematic review and meta-analysis. Appetite 125, 253-269, doi:https://doi.org/10.1016/j.appet.2018.01.018 (2018).

4 Krop, E. M., Hetherington, M. M., Holmes, M., Miquel, S. \& Sarkar, A. On relating rheology and oral tribology to sensory properties in hydrogels. Food Hydrocolloids (2018).

5 NCD Risk Factor Collaboration (NCD-RisC). Trends in adult body-mass index in 200 countries from 1975 to 2014: a pooled analysis of 1698 population-based measurement studies with 19.2 million participants. The Lancet 387, 1377-1396, doi:https://doi.org/10.1016/S0140-6736(16)30054-X (2016).

6 World Health Organization. Obesity and overweight. (World Health Organization, 2016).

7 Blundell, J. E. et al. in Handbook of Assessment Methods for Eating Behaviours and Weight-Related Problems : Measures, Theory and Research [2nd. ed.]) (eds David B. Allison \& Monica L. Baskin) 283-325 (Sage, 2009).

8 Blundell, J. E. et al. Appetite control: methodological aspects of the evaluation of foods. Obesity Reviews 11, 251-270, doi:10.1111/j.1467-789X.2010.00714.x (2010). 
9 Blundell, J. E. Pharmacological approaches to appetite suppression. Trends in pharmacological sciences 12, 147-157 (1991).

10 Hetherington, M. M. et al. Potential benefits of satiety to the consumer: scientific considerations. Nutrition research reviews 26, 22-38, doi:10.1017/s0954422413000012 (2013).

11 Pavlov, I. P. The Work of the Digestive Glands. (Charles Griffin Co Ltd, 1902).

12 Cecil, J. E., Francis, J. \& Read, N. W. Relative contributions of intestinal, gastric, orosensory influences and information to changes in appetite induced by the same liquid meal. Appetite 31, 377-390, doi:http://dx.doi.org/10.1006/appe.1998.0177 (1998).

13 Nolan, L. J. \& Hetherington, M. M. The effects of sham feeding-induced sensory specific satiation and food variety on subsequent food intake in humans. Appetite 52, 720-725, doi:http://dx.doi.org/10.1016/j.appet.2009.03.012 (2009).

14 Lavin, J. H., French, S. J., Ruxton, C. H. S. \& Read, N. W. An investigation of the role of oro-sensory stimulation in sugar satiety. International journal of obesity 26, 384-388 (2002). $\quad<\mathrm{http} / / /$ onlinelibrary.wiley.com/o/cochrane/clcentral/articles/025/CN00443025/frame.html>.

15 Hetherington, M. M. \& Boyland, E. Short-term effects of chewing gum on snack intake and appetite. Appetite 48, 397-401, doi:10.1016/j.appet.2006.10.001 (2007).

16 Hetherington, M. M. \& Regan, M. F. Effects of chewing gum on short-term appetite regulation in moderately restrained eaters. Appetite 57, 475-482 (2011).

17 Zijlstra, N., Mars, M., de Wijk, R. A., Westerterp-Plantenga, M. S. \& de Graaf, C. The effect of viscosity on ad libitum food intake. Int J Obes 32, 676-683 (2008).

18 Lasschuijt, M. P. et al. Comparison of oro-sensory exposure duration and intensity manipulations on satiation. Physiology \& Behavior, doi:http://dx.doi.org/10.1016/j.physbeh.2017.02.003 (2017).

19 Hogenkamp, P. S., Mars, M., Stafleu, A. \& de Graaf, C. Repeated consumption of a large volume of liquid and semi-solid foods increases ad libitum intake, but does not change expected satiety. Appetite 59, 419-424, doi:10.1016/j.appet.2012.06.008 (2012).

20 Zijlstra, N. et al. Effect of viscosity on appetite and gastro-intestinal hormones. Physiol Behav 97, 68-75, doi:10.1016/j.physbeh.2009.02.001 (2009).

21 Larsen, D. S., Tang, J., Ferguson, L. R. \& James, B. J. Increased textural complexity in food enhances satiation. Appetite 105, 189-194, doi:http://dx.doi.org/10.1016/j.appet.2016.05.029 (2016).

22 Stokes, J. R., Boehm, M. W. \& Baier, S. K. Oral processing, texture and mouthfeel: From rheology to tribology and beyond. Current Opinion in Colloid \& Interface Science 18, 349-359, doi:https://doi.org/10.1016/j.cocis.2013.04.010 (2013).

23 Laguna, L. \& Sarkar, A. Oral tribology: update on the relevance to study astringency in wines. Tribology - Materials, Surfaces \& Interfaces 11, 116-123, doi:10.1080/17515831.2017.1347736 (2017).

24 van Strien, T., Frijters, J. E. R., Bergers, G. P. A. \& Defares, P. B. The Dutch Eating Behavior Questionnaire (DEBQ) for assessment of restrained, emotional, and external eating behavior. International Journal of Eating Disorders 5, 295-315, 
doi:http://dx.doi.org/10.1002/1098-108X(198602)5:2<295::AID-

EAT2260050209>3.0.CO;2-T (1986).

25 Cook, S. L., Bull, S. P., Methven, L., Parker, J. K. \& Khutoryanskiy, V. V. Mucoadhesion: A food perspective. Food Hydrocolloids 72, 281-296, doi:https://doi.org/10.1016/j.foodhyd.2017.05.043 (2017).

26 Shtenberg, Y. et al. Mucoadhesive alginate pastes with embedded liposomes for local oral drug delivery. International Journal of Biological Macromolecules 111, 62-69, doi:https://doi.org/10.1016/j.ijbiomac.2017.12.137 (2018).

27 Julis, R. A. \& Mattes, R. D. Influence of sweetened chewing gum on appetite, meal patterning and energy intake. Appetite 48, 167-175, doi:10.1016/j.appet.2006.08.003 (2007).

28 Pliner, P. \& Hobden, K. Development of a scale to measure the trait of food neophobia in humans. Appetite 19, 105-120, doi:https://doi.org/10.1016/0195-6663(92)90014-W (1992).

29 Rolls, B. J. et al. Variety in a meal enhances food intake in man. Physiology \& Behavior 26, 215-221, doi:https://doi.org/10.1016/0031-9384(81)90014-7 (1981).

30 Redd, M. \& de Castro, J. M. Social facilitation of eating: effects of social instruction on food intake. Physiology \& Behavior 52, 749-754, doi:https://doi.org/10.1016/00319384(92)90409-U (1992).

31 Stone, H. \& Sidel, J. L. Sensory Evaluation Practices (Third Edition). (Academic Press, 2004).

32 Sarkar, A., Goh, K. K. T. \& Singh, H. Colloidal stability and interactions of milkprotein-stabilized emulsions in an artificial saliva. Food Hydrocolloids 23, 1270-1278, doi:http://dx.doi.org/10.1016/j.foodhyd.2008.09.008 (2009).

33 Laguna, L., Farrell, G., Bryant, M., Morina, A. \& Sarkar, A. Relating rheology and tribology of commercial dairy colloids to sensory perception. Food \& Function 8, 563 573, doi:10.1039/C6FO01010E (2017).

34 Sarkar, A., Kanti, F., Gulotta, A., Murray, B. S. \& Zhang, S. Aqueous Lubrication, Structure and Rheological Properties of Whey Protein Microgel Particles. Langmuir 33, 14699-14708, doi:10.1021/acs.langmuir.7b03627 (2017).

35 Laguna, L., Hetherington, M. M., Chen, J., Artigas, G. \& Sarkar, A. Measuring eating capability, liking and difficulty perception of older adults: A textural consideration. Food Quality and Preference 53, 47-56, doi:https://doi.org/10.1016/j.foodqual.2016.05.013 (2016).

36 Laguna, L. \& Sarkar, A. Influence of mixed gel structuring with different degrees of matrix inhomogeneity on oral residence time. Food Hydrocolloids 61, 286-299, doi:http://dx.doi.org/10.1016/j.foodhyd.2016.05.014 (2016).

37 Chen, J. \& Lolivret, L. The determining role of bolus rheology in triggering a swallowing. Food Hydrocolloids $\quad$ 25, 325-332, doi:https://doi.org/10.1016/j.foodhyd.2010.06.010 (2011).

38 Forde, C. G., Kuijk, N., Thaler, T., de Graaf, C. \& Martin, N. Oral processing characteristics of solid savoury meal components, and relationship with food composition, sensory attributes and expected satiation. Appetite 60, 208-219, doi:10.1016/j.appet.2012.09.015 (2013). 